\title{
Incidence and Pattern of Thyroid Dysfunction in Patients on Chronic Amiodarone Therapy: Experience at a Tertiary Care Centre in Oman
}

\author{
H. Farhan ${ }^{1}$, A. Albulushi*,1, A. Taqi ${ }^{2}$, A. Al-Hashim ${ }^{1}$, K. Al-Saidi ${ }^{1}$, K. Al-Rasadi ${ }^{3}$, A. Al-Mazroui ${ }^{1}$ \\ and I. Al-Zakwani ${ }^{4,5}$ \\ ${ }^{I}$ Department of Medicine, Sultan Qaboos University Hospital, Oman \\ ${ }^{2}$ Department of Pharmacy, Sultan Qaboos University Hospital, Oman \\ ${ }^{3}$ Department of Clinical Biochemistry, Sultan Qaboos University Hospital, Oman \\ ${ }^{4}$ Department of Pharmacology \& Clinical Pharmacy, College of Medicine \& Health Sciences, Sultan Qaboos Universi- \\ ty, Muscat, Oman \\ ${ }^{5}$ Gulf Health Research, Muscat, Oman
}

\begin{abstract}
Objective: To determine the incidence and pattern of thyroid dysfunction (TD) in patients on chronic amiodarone therapy.

Methods: A retrospective study which evaluated 59 patients who had received amiodarone therapy regularly for at least 12 months from a period of 3 years from October 2007 to October 2010. The patients were followed-up at the cardiac clinic at Sultan Qaboos University Hospital, Muscat, Oman.

Results: The mean age of the cohort was $63 \pm 13$ years ranging from 27 to 98 years. Fifty-one percent $(n=30)$ of the patients were female. There were $11(19 \%)$ cases of thyroid dysfunction (TD). Seven (12\%) patients were hypothyroid, 3 (5\%) had hyperthyroidism and 1 (2\%) patient had sub-clinical hypothyroidism; no cases of sub-clinical hyperthyroidism were noted. Female gender and presence of anti-thyroid peroxidase antibodies were significantly associated with amiodarone-induced hypothyroidism $(p=0.001)$ while age, amiodarone dose and duration of therapy were not correlated with the development of TD (all $p$-values $>0.05$ ).

Conclusion: Amiodarone-induced thyroid dysfunction is prevalent. Hypothyroidism was more frequent and seen more in female patients and those who had positive anti-thyroid peroxidase antibodies. Initial screening and periodic monitoring of thyroid function is mandatory for all patients on amiodarone therapy.
\end{abstract}

Keywords: Thyroid diseases, amiodarone, hypothyroidism, thyrotoxicosis, hyperthyroidism, Oman.

\section{INTRODUCTION}

Amiodarone is a potent anti-arrhythmic drug that has been widely used for the treatment and prophylaxis of various cardiac arrhythmias since its approval in 1985 by the Food and Drug Administration (FDA) [1]. Nearly 37\% of amiodarone contains organic iodine by molecular weight, so each $200 \mathrm{mg}$ tablet is estimated to contain about $75 \mathrm{mg}$ of organic iodide of which $10 \%(7.5 \mathrm{mg})$ is de-iodinated to yield free iodine. This results in a high iodine supply to the body that far exceeds the daily recommended optimal iodine intake $(0.2$ to $0.8 \mathrm{mg})[2,3]$. Moreover, the elimination halflife of amiodarone is about 2-3 months, and total body iodine stores may remain elevated for up to 9 months even after

*Address correspondence to this author at the Department of Medicine, Sultan Qaboos University Hospital, PO Box: 35: post code: 123, Al-khod, Muscat, Sultanate of Oman; Tel: +968 98517982; Fax: +968 24144430;

E-mail: dr.albulushi@gmail.com discontinuation of the drug [3]. Although amiodarone has proved to be useful, several side-effects have been associated with its chronic administration. Thyroid dysfunction (TD) is considered to be a common and potentially serious sideeffect [4]. Amiodarone-induced thyroid dysfunction (AITD) is a complex phenomenon; however, its exact pathogenesis largely remains unknown [5].

Amiodarone-induced hypothyroidism (AIH) is considered to be mediated by excess iodine. The large amount of iodine released by the metabolism of amiodarone intake results in an adaptive block of further intra-thyroidal iodide uptake and hence inhibits thyroid hormone synthesis, the so called Wolff-Chaikoff effect [6]. Escape from the WolffChaikoff effect usually occurs even with the continued use of amiodarone and further exposure to high iodine and this prevents hypothyroidism [7]. Failure of the thyroid to escape from the Wolff-Chaikoff effect causes persistent blockage in the intra-thyroidal iodide oxidation and decreased thyroid hormone synthesis consequently leading to hypothyroidism 
$[8,9]$. Moreover, a defect that results in enhanced susceptibility to the inhibitory effect of iodine on thyroid hormone synthesis has also been documented [10].

The factors leading to the development of thyrotoxicosis in patients treated with amiodarone are not completely understood. Two forms of amiodarone-induced thyrotoxicosis (AIT) have been described $[11,12]$. Type I is caused by iodine induced excess thyroid hormone synthesis and release especially in patients with an underlying thyroid disorders such as multinodular goiter or Graves' disease [11]. Type II usually occurs in those patients with previously normal thyroid gland and is caused by direct cytotoxic effect of amiodarone on thyroid follicles and release of preformed thyroid hormones. However, mixed forms of AIT may occur in an abnormal thyroid gland [12]. Minor derangements in thyroid function tests have also been known to occur with amiodarone therapy but these are of no clinical significance and do not require specific treatment.

The exact incidences of (AIT) and (AIH) are unknown but the reported incidence of AIT ranges from 1.4 to $21 \%$ and that of AIH from 2 to 27\%; the overall incidence of AITD has been reported to be around 2 to $24 \%[13,14]$ However, studies on the incidence of AIT and AIH in Oman or the Arabian Gulf region are scant. Hence, we assessed the incidence and pattern of thyroid dysfunction at Sultan Qaboos University hospital (SQUH) in Oman.

\section{METHODS}

This was a retrospective study conducted over a 3-year period from October 2007 to October 2010, at a cardiology clinic at SQUH. The hospital has computerized medical record for all patients. The medical record numbers of all patients who received regular amiodarone therapy were obtained from the computerized hospital pharmacy record. The study included all adult ( $\geq 18$ years) patients who received amiodarone therapy for at least 1 year during the study period.

Variables, like age, gender, indications of amiodarone therapy, dose and duration, and results of thyroid functions tests were elicited from the computerized hospital information system. The serum samples for thyroid stimulating hormone (TSH), free thyroxine (FT4), free triiodothyronine (FT3) were measured using automated Chemiluninicus assays manufactured by Bayer (Access 2, Beckman Coulter, Galway, Ireland). Thyroid functions tests were assessed at baseline i.e. before the start of amiodarone therapy, at 3 months and then every 6 months over the specified period of study.

The incidence and pattern of thyroid dysfunction was based exclusively on laboratory diagnostic criteria. Patients were diagnosed to have hyperthyroidism when suppressed TSH (<0.34 mIU/L; reference range: 0.34-5.60 mIU/L) was found in combination with an elevated FT4 (>14.4 pmol/L; reference range: $7.9-14.4 \mathrm{pmol} / \mathrm{L})$ and/or FT3 (>6.0 pmol/L; 3.8-6.0 pmol/L). Elevated FT4 (>14.4 pmol/L) alone was not considered diagnostic of hyperthyroidism. Patients with suppressed TSH $(<0.34 \mathrm{mIU} / \mathrm{L})$ and normal FT4 (7.9-14.4 $\mathrm{pmol} / \mathrm{L})$ and FT3 (3.8-6.0 pmol/L) were considered to have sub-clinical hyperthyroidism. Hypothyroidism was diag- nosed if TSH was elevated (>5.6 mIU/L) and FT4 reduced ( $<7.9 \mathrm{pmol} / \mathrm{L})$, while those with elevated TSH $(>5.6 \mathrm{mIU} / \mathrm{L})$ and normal FT4 (7.9-14.4 pmol/L) were considered to have sub-clinical hypothyroidism. Thyroid anti-bodies (Euroimmun, Lebeck, Germany) were not checked routinely before the start of amiodarone therapy. In fact, anti-thyroid peroxidase antibodies (Anti-TPO) were ordered only in those patients who had biochemical evidence of clinical or subclinical hypothyroidism. They were also only ordered at baseline and not at follow-up.

\section{Statistical Analysis}

Descriptive statistics were used to describe the data. For categorical variables, frequencies and percentages were reported. Differences between groups were analyzed using Pearson's chi-squared tests (or Fisher's exact tests for cells < $5)$. For continuous variables, means and standard deviations were used to summarize the data, while analyses were performed using Student's t-test or univariate linear ordinary least squares (OLS) regression, wherever appropriate. A 2 tailed level of significance was set at $p=0.05$. All statistical procedures were performed using STATA version 12.1 (College Station, Texas, USA).

\section{RESULTS}

A total of 59 patients who fulfilled the inclusion criteria were enrolled into the study. The overall mean age of the cohort was $63 \pm 13$ years ranging from 27 to 98 years; $51 \%$ $(\mathrm{n}=30)$ of the patients were females. The overall mean \pm SD duration of amiodarone therapy in all study patients was $21 \pm$ 7 months (ranging from 12 to 36 months). The underlying cardiac indications for which amiodarone therapy was started are shown in Table 1. Atrial fibrillation (AF) was the most common type of arrhythmia observed $(53 \%$; $n=31)$. Cardiomyopathy of any etiology (20\%), supra-ventricular tachycardia (SVT) (15\%) and ventricular tachycardia (VT) (12\%) were among the other indications.

The incidence and pattern of thyroid dysfunction in patients on amiodarone therapy are shown in Table 2. Among the 59 study patients, 11 (19\%) patients developed any kind of new onset thyroid dysfunction. Seven patients (12\%) developed hypothyroidism, 1 (2\%) had sub-clinical hypothyroidism while $3(5 \%)$ had hyperthyroidism. Forty-eight $(81 \%)$ patients remained euthyroid. In 48 euthyroid patients, 2 (4\%) had isolated high FT4 levels (>14.4 pmol/L).

The patients were grouped in into 3 categories (Table $\mathbf{3}$ ). One sub-clinical hypothyroid patient was included in the

Table 1. Indications for Amiodarone Therapy in the Study Patients $(\mathbf{n}=59)$

\begin{tabular}{|l|l|}
\hline \multicolumn{1}{|c|}{ Indication/Cardiac Diagnosis } & \multicolumn{1}{c|}{ Number of Patients (\%) } \\
\hline \hline Atrial fibrillation & $31(53 \%)$ \\
\hline Cardiomyopathy & $12(20 \%)$ \\
\hline Supra-ventricular tachycardia & $9(15 \%)$ \\
\hline Ventricular tachycardia & $7(12 \%)$ \\
\hline
\end{tabular}


Table 2. Incidence and Pattern of Thyroid Dysfunction in Patients on Amiodarone Therapy $(n=59)$

\begin{tabular}{|l|l|}
\hline \multicolumn{1}{|c|}{ Thyroid Status } & \multicolumn{1}{c|}{ Number of Patients (\%) } \\
\hline \hline Euthyroid & $48(81 \%)$ \\
\hline Hypothyroid & $7(12 \%)$ \\
\hline Sub-clinical hypothyroid & $1(2 \%)$ \\
\hline Hyperthyroidism & $3(5 \%)$ \\
\hline All new thyroid dysfunction & $11(19 \%)$ \\
\hline
\end{tabular}

hypothyroid group for analysis. There were no significant differences in age in patients who remained euthyroid compared with those who were either hypothyroid or hyperthyroid (22 vs 19 vs 24 years; $p=0.421$ ). Among 11 patients with thyroid dysfunction, 7 (64\%) were female. Moreover, it was also noted that majority of the patients in the hypothyroid group were female (75\%) compared to euthyroid (42\%) and hyperthyroid $(33 \%)$ groups $(p=0.010)$. Although the duration of treatment was slightly higher in patients who develop hyperthyroidism ( $24 \pm 6$ months) as compared with the hypothyroid $(19 \pm 7$ months) and euthyroid $(22 \pm 7$ months) patients, the results were not significant $(p=0.467)$.

The majority of our patients received $200 \mathrm{mg} / \mathrm{day}$ of amiodarone as maintenance dose and therefore, the association between various amiodarone doses and the development of new thyroid dysfunction was not possible. Among 7 hypothyroid patients, $5(71 \%)$ had positive anti-thyroid antibodies. Among the 5 anti-TPO positive patients, $4(80 \%)$ were female. Development of new thyroid dysfunction was not related to age and duration of amiodarone therapy (all $p>$ 0.05). As expected, there were significant differences in TSH and FT4 in hypothyroid and hyperthyroid group compared with the euthyroid patients $(p=0.001)$.

\section{DISCUSSION}

This study has highlighted the significance occurrence of thyroid dysfunction due to amiodarone therapy in the region, which were mostly hypothyroidism rather than hyperthyroidism. To our knowledge this is the first study not only in Oman but the Arabian Gulf at large.
It has been demonstrated that during the early (usually the first 3) months of amiodarone therapy, transient and clinically non-significant thyroid function abnormalities are common and do not need specific treatment $[2,15]$. However, the current study only analyzed those patients that received amiodarone therapy regularly for at least 12 months. The high incidence of TD reported in the current study was not attributed to such transient abnormalities. Most of the patients in the current study were old and had ischemic heart disease. However, age was not associated to the development of TD in the study group and similar findings of no correlation between age and the development of AITD have also been reported [16].

It has been described that regional differences may influence the incidence and pattern of thyroid dysfunction due to amiodarone therapy [17]. In those areas where iodine intake is sufficient, hypothyroidism has been reported as the most common amiodarone-induced thyroid dysfunction (AITD) ranging from 13 to $25 \%[17,18]$. However, the occurrence of hyperthyroidism is mainly seen in those areas where iodine intake is low [19].

The results of our study indicate an incidence of $19 \%$ of AITD due to chronic amiodarone therapy which is comparable to previous studies [14, 16, 20]. Hypothyroidism (clinical and sub-clinical) was the most common type of thyroid dysfunction seen in $12 \%$ of our patients. One of the factors which may explain this observation is that the cohorts of our study patients may have sufficient iodine intake due to the Royal Decree in 1995 for universal salt iodization in the Sultanate of Oman [21-23]. Some other studies conducted in iodine sufficient areas have shown low incidence of hypothyroidism and did not support the general agreement that AIH is linked with iodine intake; however, it is also postulated that there may be mechanisms other than iodine which might play a role in the development of AIH [15, 24].

The majority of the patients who developed hypothyroidism were female and had positive anti-TPO. This could be explained by the fact that women usually have greater propensity to develop autoimmune thyroid diseases like Graves disease and Hashimoto's thyroiditis and amiodarone can modify the natural course of such pre-existing diseases [25]. Our study included only patients who were euthyroid before starting amiodarone therapy and thyroid antibodies were not checked at the beginning of treatment. Therefore, the detec-

Table 3. Comparison of on Clinical Data of Studied Patients on Basis of Thyroid Function $(n=59)$

\begin{tabular}{|l|l|l|l|l|}
\hline \multicolumn{1}{|c|}{ Characteristic } & Euthyroid, $\mathbf{n = 4 8}(\mathbf{8 1 \%})$ & Hypothyroid, $\mathbf{n = 8}(\mathbf{1 4 \%})$ & Hyperthyroid, $\mathbf{n = 3}(\mathbf{5 \% )}$ & \multicolumn{1}{c|}{$\boldsymbol{p}$} \\
\hline \hline $\begin{array}{l}\text { Male, } \mathrm{n}(\%) \\
\text { Female, } \mathrm{n}(\%)\end{array}$ & $\begin{array}{l}28(58 \%) \\
20(42 \%)\end{array}$ & $\begin{array}{l}2(25 \%) \\
6(75 \%)\end{array}$ & $\begin{array}{l}2(67 \%) \\
1(33 \%)\end{array}$ & 0.010 \\
\hline Age, mean \pm SD, (years) & $62 \pm 14$ & $65 \pm 8$ & $70 \pm 6$ & 0.421 \\
\hline $\begin{array}{l}\text { Treatment duration, } \\
\text { (months) }\end{array}$ & $22 \pm 7$ & $19 \pm 7$ & $24 \pm 6$ & 0.467 \\
\hline FT4, mean \pm SD, pmol/L & $12.01 \pm 1.502$ & $6.25 \pm 1.66$ & $20.25 \pm 2.21$ & 0.001 \\
\hline TSH, mean \pm SD, mIU/L & $2.29 \pm 0.795$ & $13.27 \pm 5.51$ & $0.20 \pm 0.12$ & 0.001 \\
\hline
\end{tabular}

SD, standard deviation; FT4, free thyroxine; TSH, thyroid stimulating hormone 
tion anti-TPO in hypothyroid patients raises the possibility that chronic use of amiodarone may have unmasked preexisting sub-clinical autoimmune hypothyroid disease in some patients. The increased association between AIH and female gender has been well described [17]. The risk of development of hypothyroidism associated with amiodarone is 13 times greater in female patients who were positive for AntiTPO as compared with men without thyroid antibodies [5, 15].

As previously mentioned, AIT is more commonly observed in areas of low iodine intake [17, 27]. The reported incidence of AIT in iodine sufficient or replete areas is about $2 \%$ while in iodine depleted areas is it is $24 \%$ or even higher $[17,26]$. The current study reports AIT in $5 \%$ of the patients, which is slightly higher, considering that the region is iodine sufficient. However high incidence of AIT has been reported in certain studies conducted in areas of iodine sufficiency and had led to concerns that factors other than iodine exposure may be responsible in the pathogenesis and development of AIT $[5,16]$. The current study did not measure thyroid antibodies in patients with hyperthyroidism, and moreover due to the retrospective nature of our study we could not find other factors in association with AIT. Hence, it is clear that thyrotoxicosis in our patients was a result of type I or type II or combination of both.

The current study could not find an association between TD and duration of amiodarone therapy. A relationship between amiodarone dose and the development of thyroid dysfunction could not be measured due to the fact that almost all of the patients, whether euthyroid or had TD, were on a daily dose of $200 \mathrm{mg}$. This is in agreement with the previous reports that could not demonstrate an association between the duration of amiodarone treatment and TD which also used the small dose $(200 \mathrm{mg} /$ day $)$ of amiodarone in the majority of patients [17, 27].

The management of AIT is more difficult and challenging than $\mathrm{AIH}$ and may warrant withdrawal of amiodarone. Even if the drug is discontinued, thyrotoxicosis may take as long as 8 months to subside by virtue of the prolonged halflife of amiodarone. Treatment options include thionamides, with or without potassium perchlorate, depending upon the severity of thyrotoxicosis, while steroids have also proven useful especially in type II AIT [28-30]. AIH is usually managed either by discontinuation of amiodarone therapy or thyroid hormone replacement or both [31-33]. The few patients in the current study were affectively treated with anti-thyroid drugs, while hypothyroid patients were treated with thyroxine replacement and amiodarone was continued. Despite the controversy [34], the one patient with sub-clinical hypothyroidism in this study was also treated with low dose levothyroxine ( $25 \mu \mathrm{g}$ daily). Evidence suggests that the addition of levothyroxine may exert a beneficial effect on the lipid profile [35]. Furthermore, it is also postulated that the treatment may also improve some other markers associated with cardiovascular disease such as carotid intima media thickness (cIMT) indices of endothelial function and other predictors of vascular risk [35]. However, the metabolic and cardiovascular bio-makers were, unfortunately, not measured in the patient with sub-clinical hypothyroidism.
This study is not without limitations. Due to its retrospective nature, we could not evaluate relationships between clinical and laboratory diagnosis of TD. This study relied only on biochemical diagnosis. The association between amiodarone and thyroid dysfunction would have been strengthened even further had the study included a matched control group, not on amiodarone therapy. However, this bias is mitigated by the previous published studies on the positive correlation between amiodarone and thyroid dysfunction. Furthermore, the study was also low powered in some aspects due its small sample size.

\section{CONCLUSIONS}

Amiodarone-induced thyroid dysfunction (AITD) is prevalent. Hypothyroidism was more frequent and seen more in female patients and those who had positive anti-thyroid peroxidase antibodies. Initial screening and periodic monitoring of thyroid function should be mandatory in all patients on amiodarone therapy. Furthermore, larger prospective cohort studies are warranted to validate our findings.

\section{DECLARATION OF CONFLICTING INTERESTS}

The authors declare no conflicting interest with respect to the authorship and/or publication of this manuscript.

\section{FUNDING}

The authors received no financial support for the research, authorship and/or publication of this article.

\section{REFERENCES}

[1] Reiffel JA, Estes NA 3rd, Waldo AL, Prystowsky EN, DiBianco R. A consensus report on antiarrhythmic drug use. Clin Cardiol 1994; 17: 103-16.

[2] Harjai KJ, Licata AA. Effects of amiodarone on thyroid function. Ann Intern Med 1997; 126: 63-73.

[3] Fradkin JE, Wolff J. Iodide-induced thyrotoxicosis. Medicine (Baltimore) 1983; 62: 1-20.

[4] Vorperian VR, Havighurst TC, Miller S, January CT. Adverse effects of low dose amiodarone: a meta-analysis. J Am Coll Cardiol 1997; 30: 791-798.

[5] Thorne SA, Barnes I, Cullinan P, Somerville J. Amiodaroneassociated thyroid dysfunction: risk factors in adults with congenital heart disease. Circulation 1999; 100: 149-54.

[6] Wolff J, Chaikoff L. Plasma inorganic iodiode as a homeostatic regulator of thyroid function. J Biol Chem 1948; 174: 555-60.

[7] Selenkow HA, Garcia AM, Bradley EB. An autoregulatory effect of iodide in diverse thyroid disorders. Ann Intern Med 1965; 62: 714-26.

[8] Martino E, Aghini-Lombardi F, Mariotti S, et al. Amiodarone iodine-induced hypothyroidism: risk factors and follow-up in 28 cases. Clin Endocrinol (Oxf) 1987; 26: 227-37.

[9] Martino E, Bartalena L,Mariotti S, et al. Radioactive iodine thyroid uptake in patients with amiodarone-iodine-induced thyroid dysfunction. Acta Endocrinol 1988; 119: 167-73.

[10] Figge HL, Figge J. The effects of amiodarone on thyroid hormone function: a review of physiology and clinical manifestations. J Clin Pharmacol 1990; 30: 588-95.

[11] Bartalena L, Brognioni S, Grasso L, Bogazzi F, Burelli A, Martino E. Treatment of amiodarone induced thyrotoxicosis, a difficult challenge: results of a retrospective study. J Clin Endocrinol Metab 1996; 81: 2930-3.

[12] Klein I, Ojamaa K. Thyroid hormone and the cardiovascular system. N Engl J Med 2001; 344: 501-9.

[13] Bogazzi F, Tomisti L, Bartalena L, Aghini-Lombardi F, Martino E. Amiodarone and the thyroid: a 2012 update. J Endocrinol Invest 2012; 35: 340-8. 
[14] Serdiuk SE, Bakalov SA, Golitsyn SP, Molashenko NV, Platonova NM, Sviridenko NIu. Incidence and predictors of thyroid dysfunction caused by long-term intake of amiodarone. Ter Arkh 2005; 77: $33-9$.

[15] Trip MD, Wiersinga W, Plomp TA. Incidence, predictability, and pathogenesis of amiodarone-induced thyrotoxicosis and hypothyroidism. Am J Med 1991; 91: 507-11.

[16] Ross IL, Marshall D, Okreglicki A, Isaacs S, Levitt NS. Amiodarone-induced thyroid dysfunction. S Afr Med J 2005; 95: 180-3.

[17] Trifănescu R, Fica S, Barbu C, et al. Amiodarone-induced thyroid dysfunction in cardiac patients from areas with iodine deficiency. Rom J Intern Med 2004; 42: 595-605.

[18] Schaan BD, Cunha CP, Francisconi A, et al. Amiodarone-induced thyroid dysfunction in a tertiary center in south Brazil. Arq Bras Endocrinol Metabol 2005; 49: 916-22.

[19] Toni M, Anda E, Pineda J, Martínez de Esteban JP, Ollero MD. The effects of amiodarone on the thyroid. An Sist Sanit Navar 2009; 32: 363-70.

[20] Tsadok MA, Jackevicius CA, Rahme E, et al. Amiodarone-induced thyroid dysfunction: brand-name versus generic formulations. CMAJ 2011; 183: E817-23.

[21] Kapil U. Health consequences of iodine deficiency. Sultan Qaboos Univ Med J 2007; 7: 267-72.

[22] Monitoring of Universal salt iodization: a collaborative project of MOH/UNICEF. Ministry of Health, Muscat, 1998.

[23] Ministry of Health, UNICEF. Monitoring Oman's Progress in Achieving Universal Salt Iodization. MOH/UNICEF-Oman, 2000.

[24] Sato K, Miyakawa M, Eto M, et al. Clinical characteristics of amiodarone-induced thyrotoxicosis and hypothyroidism in Japan. Endocr J 1999; 46: 443-51.

[25] Martino E, Aghini-Lombardi F, Bartalena L, et al. Enhanced susceptibility to amiodarone-induced hypothyroidism in patients with thyroid autoimmune disease. Ann Intern Med 1994; 154: 2722-6.
[26] Martino E, Safran M, Aghini-Lombardi F, et al. Environmental iodine intake and thyroid dysfunction during chronic amiodarone therapy. Ann Intern Med 1984; 101: 28-34.

[27] Pazin-Filho A, de Jesus AM, Magalhães PK, et al. How frequently should a patient taking amiodarone be screened for thyroid dysfunction. Braz J Med Biol Res 2009; 42:744-9.

[28] Padmanabhan H. Amiodarone and thyroid dysfunction. South Med J 2010; 103: 922-30.

[29] Martino E, Aghini-Lambardi F,Mariotti S, et al. Treatment of amiodarone associated thyrotoxicosis by simultaneous administration of potassium perchlorate and methimazole. J Endocrinol Invest 1986; 9: 201-7.

[30] Broussolle C, Ducottet X, Martin C, et al. Rapid effectiveness of prednisone and thionamides combined therapy in severe amiodarone iodine-induced thyrotoxicosis: comparison of two groups of patients with apparently normal glands. J Endocrinol Invest $1989 ; 12: 37-42$.

[31] Bednarek-Tupikowska G, Filus A, Kuliczkowska J, Bugajski J. Amiodarone and the thyroid gland. Postepy Hig Med Dosw (Online) 2004; 58: 216-25.

[32] Nademanee K, Piwonka RW, Singh BN, Hershman JM. Amiodarone and thyroid function. Prog Cardiovasc Dis 1989; 31: 427-37.

[33] Brogioni S, Dell'Unto E, Cosci C, et al. Amiodarone-Induced Thyrotoxicosis. Int J Endocrinol 2006; 4: 52-62.

[34] Surks MI, Ortiz E, Daniels GH, et al. Subclinical thyroid disease: scientific review and guidelines for diagnosis and management. JAMA 2004; 291: 228-38.

[35] Lioudaki E, Mavroeidi NG, Mikhailidis DP, Ganotakis ES. Subclinical hypothyroidism and vascular risk: An update. Hormones 2013. In press

Received: November 01, 2013

Accepted: November 04, 2013

(C) Farhan et al.; Licensee Bentham Open.

This is an open access article licensed under the terms of the Creative Commons Attribution Non-Commercial License (http://creativecommons.org/licenses/ by-nc/3.0/) which permits unrestricted, non-commercial use, distribution and reproduction in any medium, provided the work is properly cited. 